\title{
TRATAMIENTO QUIRURGICO DEL EMPIEMA EN EL NIÑO
}

\author{
Por el Dr. AgUSTIN INOSTROSA P.
}

Jefe Sección Cirugía del Hospitol Mannel Arriarán.

(Relator oficial sobre el tema: Tratamiento médico-quizirgico del empiema
en 1 niño).

El tema que tengo el honor de relatar se refiere al tratamiento quirúrgico del Empiema en los niños. Su estudio ha sido ampliamente abordado en diversos Congresos tanto nacionales como extranjeros. En línea generales se puede decir que en ellos se ha llegado a conclusiones más o menos semejantes y que si bien es cirto. no han logrado hasta ahora. dar una pauta definitiva para su curación, a lo menos han permitido formarse un concepto en culanto a la evolución y scbre todo en lo que a su tratamiento se refiere.

A pesar de lo dicho, siempre es un tema de actualidad. siendo a menudo discutido, a lo menos entre nosotros dado el gran número de casos que se presentan en nuestros Servicios.

Es por esto, que al desartollar nuestro trabajo, pasaremos en revista muy rápidamente el cuadro clínico así como las diversas téenicas empleadas en el tratamiento quitúrgico, limitándonos por sobre todo a un estudio estadístico a base da las observaciones recogidas en el Hospital de niños "Manuel Arriarán" y comprendidas entre los años 1930 y 1940 inclusives. Su número alcanza a 459 casos, de los cuales fueron ttaiados quirúrgicamente en el Servitis a mi cargo 371 . siendo el resto, o sea 88 oasos tratados médicamente.

Como se comprende los poncentajes que expondremos más adelante no pueden tener valor abșoluto, puesto que un gran número de factores contribuyen a modificarlos. tales como la edad, los caracteres epidemiológicos de un año a otro, las estaciones del año, las eventualidades post-operatorias, etc. 
Así por ejemplo, tenemos que su observación es más frecuente en el invierno y en la primavera, donde henos tratado 125 y 90 respectivamente, en tanto que en el verano tenemos 75 y en el otoño 80 casos.

En cuanto al sexo se refiere, podemos decir que el masculino da un mayor número de casos, 221, mientras que el femenino sólo alcanza a 150 ,

Respecto a la localización de la afección, podemos afirmar que hay una marcada predilección por el lado derecho, 264, mientras que sólo 195 corresponden al lado izquierdo. Ld localización bilateral ha sido observada en siete casos de los cuales fallecieron cuatro.

Sin duda que la edad es un factor de la más alta importancia, en cuanto al pronóstico se refiere. La mayor parte de las estadísticas publicadas hacen dos agrupaciones: una de los casos hasta los dos años y otra de las observaciones sobre ésa edad.

En nuestras observaciones. el primer grupo, o sea hasta los dos años, comprende 103 casos, de los cuales fallecieron 37. lo que da una mortalidad operatoria hasta los dos años de un $35,92 \%$ y de $64,08 \%$ de curaciones. Puedo señalar la rara coincidencia de que estas icifras son casi exactamente en forma inversa, las mismas que señalla el Cirujano Ruiz Moreno en interesante y acabada comunicación al 7.9 Congreso Anual de lia Asociación Argentina de Cirugía en 1935, sobre empiema en el niño, quien sobre 51 casos operados da un porcentaje de $35 \%$ de éxitos contra $65 \%$ de decesos.

El $2^{\circ}$. grupo, sobre los dos años, comprende 268 casos, de los cuales fallecieron 44 , lo que da un $16.41 \%$ de mortalidad y un $83.59 \%$ de éxitos.

Abcra bien, tomando el porcentaje de la totalidad de nuestros operażos, o sea sobre 371 , tenemos un $22,64 \%$ de mortalidad operatoria y un $77,35 \%$ de curaciones.

Estas cifras, que aunque no marcan un desideratum en cuanto a los resultados operatorios, son bastantes satisfactorios, más aún si las comparamos con las señaladas en las estadisticas extranjeras: Yohrer, de New York, sobre un total de 265 casos, da una mortalidad de $40 \%$ de 0 a 1 año y de 28 \% hasta dos años.

Brawn, de Alemania, sobre una estadística de 125 casos da una mortalidad global de $28 \%$.

Por nuestra parte consideramos, sobre todo estudiando el detalle de nuestras estadisticas, que los resultados alcanzados 
son debidos, en parte, al concepto establecido en nuestro Hospital de no seguir insistiendo por mucho tiempo punciones terapéuticas.

En los primeros años recibiamos nuestros pacientes en condiciones un tanto desfavorables debido al agotamiento de los enfermitos por una evolución más o menos prolongada.

Así por ejemplo hacia el año 1931 tenemos una mortalidad operatoria de un $46,66 \%$, en tanto que en los últimos años en que los enfermos han sido operados en forma precoz, aurique siempre retardada, los porcentajes de éxitos han mejorado en forma manifiesta. En el año 1937 tenemos un $15.62 \%$ de mortalidad, en el año 1938 un $10.20 \%$ en 1939, $8.77 \%$ y en 1940 apenas un $8.33 \%$.

Sin duda que han contribuido a estos mejores resultados la práctica establecida en los últimos años de poder disponer en la Sección Quirúrgica de un asesor médico, en forma de que actúan en el post-operatorio, tanto el médico como el Círujanto.

Por último debo insistit en un thecho, que a mi juicio es de la más alta importancia en los resultados finales, o sea en el cuidado más prolijo de las curaciones cualquieta que sea el procedimiento empleado. Siempre éstas deben ser efectuadas por el cirujano tratante o a lo menos por un personal de enfermeras perfectamente entrenadas y conocedoras de los peligros que encierra cualquier descuido de curaciones.

En cuanto a los gérmenes encontrados en el pus podemos decir que alrededor de un $70 \%$ corresponde a pneumococisus siendo en seguida los más frecuentemente observados los estaphilococcus, los estreptococcus, y el bacilo de Pffeifer y por úlimo el colibacilo.

Generalmente en el niño menor la afección pulmonar que se complica de empiema, es la broncopneumonia en tanto que al niño mayor lo son las pneumonias. En la inmensa mayoría de los casos tienen un carácter metapneumónico.

La punción plueral, aunque no indispensable para el diagnóstico de un empiema, tiene por sobre todo su valor para demostrar la naturaleza del derrame. Al mismo tiempo nos permite apreciar el momento evolutivo de la enfermedad. Según los datos suministrados podemos decidir una intervençión inmediata o retardarla. Si encontramos, por ejemplo, streptococcus o un derrame pútrido, la intervención se hará tan pronto como sea posible. Si hay pneumocoscus y sobre todo, si el 
líquido purulento aparece algo claro. nos permitirá esperar algún tiempo.

La punción siempre es inocua, pero a condición de que sea practicada por un personal experimentado. Estimamos que en lo posible debe ser efectuada por un cirujano. Como sitio de elección se señala la región en que la matidez hídrica es más intensa, coincidiendo con los signos auscriltatórios.

Algunos autores han señalado colapsos bruscos mortäles. No los hemos observado en nuestra práctica. En cambio hemos tenido oportunidad de ver cuadros gravísimos, uno de cllos mortal, en casos de empiema de lactantes en los cuales se han practicado punciones evacuadoras abundantes, tratando de hacer un vaciamiento máximo. Estos accidentes, hoy dia perfectamente conocidos, sabemos que son debidos a la inestabiiidad mediastinal o trepidación mediastinal, motivadas por el violento cambio de la presión intrapleural.

Con todo esto se ha concluido que las punciones en el nlnio menot, sobre todo las punciones precoces deben ser pequeñas, esto es, extraer 10 a $15 \mathrm{cc}$. la primera vez, para continuar en los días siguientes con punciones mayores.

Una importancia enormé tiene el momento evolutivo de la enfermedad para decidir un determinado tratamiento. Siendo ei principal agente el pneumococcus, que determina casi siempre infecciones icon evolución más o menos típicas tenemos que el tratamiento cruento no debe ser empleado sino cuando la algidez del proceso ha declinado, haciendo por lo tanto una pleurotomia retardada.

En nuestro servicio este problema de la espera, muy pocas veces hemos tenido que resolverlo. Generalmente nos llegan los niños con una evolución más o menos larga, sea porque su ingreso al Hospital ha sido tardío o sea porque ya han sido observados o tratados en los servicios de Medicina.

En cuanto al tratamiento quirúrgico mucho se ha discutido sobre las ventajas del tórax cerrado a abierto. Como todos sabemos, el drenaje a tórax abierto es aquel que se hace abriendo la pleura $\mathrm{v}$ colocando drenajes que junto con permitir la salida de pus al exterior, permiten una libre entrada del aire exterior. En cambio en el drenaje a tórax cerrado se emplean dispositivos a métodos especiales que facilitan la salida del pus, impidiendo que el aire exterior pueda entrar a la caridad.

Las objeciones que se hacen al tórax abierto se basan en las alteraciones que determinan la desaparición de la presión 
pleural negativa y sobre todo a lo imposibilidad que esto acarteła para una libre expansión del pulmón que hasta ese momento se encuentra comprimido por el derrame.

Segün algunos autores estos inconvenientes desaparecen con el empleo del tórax cerrado facilitando por otra parte el acercamiento a la plared costal y la formación de adherencias.

La experiencia recogida en nuestro Servicio nos permite decir, que las dos técnioas tienen sus indicaciones especiales y que muy frecuentemente se emplean en forma sucesiva ambos procedimientos.

Empleamos de preferencia el drenaje cerrado en los niños menores. A este propósito debo señalar el becho de que en estos casos nos valemos'en form'a sistemática de una simple pleurotomía mínima intercosttal.

Por 10 que a los'niños mayores se refiere, cualquiera que sean los drenajes empleados los colocamos después de haber practicado la resección costal. De acuerdo con los casos de osteitis coscales observadas en el servicio y que prolongan por mucho tiempo fístulas operatorias, creemos que el cirujano al practicar la resección debe cuidar de desperiosiar la costilla sólo en la parte que se propone resecar. El proceder de otro modo expone a la aparición de procesos de osteitis.

Sin desear entrar en detalles de técnica que no tienen mayor importancia en esta comunicacíán, estimo necesario hacer presente, el buen resultado que nos ba dado el empleo de la incisión vertical de las partes blandas que permite un amplio campo para las resecciones posteriores, sobre todo cuando no se ha alcanzado la costilla precisa.

Los drenjajes pueden retirarse cuando el pulmón se ha distensido, lo que es posible comprobarlo clínica y radiológicamente; 'cuando el pus se ha hecho escaso y fluido y por último cuando el drenaje se ha obstruido.

En todas nuestras intervenciones hemos empleado indistintamente, tanto la anestesia general como la local. Le damos preferencia en el niño menor a la general, pues nos permite actuar más rapidamente y con una anestesia mínima que no tiene mayores inconvenientes. En cambio en el niño mayor, debe emplearse la anestesia local.

El término medio de tiempo de curación total es aproximadamente de 70 días.

Como complicaciones hemos observado las osteitis costales, los empiemas ctónicos con engrosamiento pleural. $\mathrm{Mu}$ chos de estos casos han obligado a practicar resenciones o toracoplástías. Muy raramente hemos observado escoliosis que 
traducen la posición habitual del enfer:no durante la evolvción de la enfermedad.

Como tratamiento complementario del médico que pasamos por alto, le damos gran importancia a los ajercicios respitatorios. En el niño menor al gateo o reptación. como también a la suspensión que permita iniprimir movimientos de balance.

\section{Conclusiones.}

1."- El empiema pleural es más frecuente en el sexo mascalino que en el femenino.

2.9- Una mayor frecuencia se observa en el invierno, después en la primavera, enseguida en el otoño y por último en el verano.

3. - Existe una marcada predilección por su localiza. ción en el tórax derecho.

4. - Como etimología tenemos en primer lugar las neumonias y enseguida las bronconeumonias.

5. ${ }^{9}$ - Los agentes microbianos más frecuentemente $a b$ servades son primero los proumocockus, después los staphilococcus. los streptococcus, el Bacilo de Pfféifer y el coli bacilo.

6. - La mayor mortalidad operatoria se observa en el riño hasta los dos años, donde alcanza un porcen-

taje de $35,92 \%$.

7 - Los empiemas tratados con punciones evacuadoras, seguidas o no de líquidos modificadores mejoran buen número de casos.

8. - Los porcentajes globales de curaciones son mayores con el tratamiento quirúrgico.

9. - E1 tratamiento más recomendable será el de punciones progresivas, durante 10 a 15 dias: después toracotomias cerradas, seguida de toracotomía abierta, hasta el término de la curación.

10.\% - Ocupan un lugar preponderante en los resultados finales, el tratamiento médico general, como así mismo el cuidado prolijo de las curaciones posteriores 